riture se trouvant auprès d'elles. Il semble qu'il en soit ainsi dans uI certain nombre de cas : les Salmonidés (Truite, Saumon), le Brochet, ont une area plus perfectionnée qu'une Carpe, qu'une 'Tanche, 'qu un Pleuronecte. Mais il existe des espèces carnassières : le Congre, I'Inguille, le Poisson-Chat, sûres et rapides dans la poursuite de leur proie, qui possèdent une mauvaise réline. Entiı, l'Hippocampe qui possède la fovea la meilleure que l'on connaisse chez les Poissons, n'éveille, en aucune manière, l’idée d'une bête de proie. Les mèmes remarques pourraient être faites en ce qui concerne l'élendue du champ de vision binoculaire : lAnguille, le Congre, le Poisson-Chat, aussi carnassiers et rapides que la Truite ou le Brochel, possèdent des champs de vision bien plus réduits.

Ainsi, nous avons vu que l'ail des Poissons est un organe imparfait ; nous constatons qu'il n'existe aucune règle précise entre les variations de structure et de fonctionnement de cet organe et l'habitat et le comportement des différentes espèces. Nous ne saurions retrowier chez les Poissons les adaptations nettes au mode de vie que l'on a décrites chez certains Reptiles, chez les ()iseaux et les Mammifères diurnes et nocturnes. Che $z$ ces derniers, le comportement paraît ètre à dominance visuelle. Il n'en n'est pas ainsi chez les Poissons. Il serait alors intéressant de connaître quel rôle les autres facultés sensorielles : gout et sens vibratoire, peuvent jouer dans le déterminisme des réactions de ces derniers ( 1 ).

\title{
L'EXPOSITION DE PISCICULTURE DE PARIS EN DÉCEMBRE 1928
}

\author{
Par M. Alfred AllotTe \\ Conservatcur des Eaux et Forêts \\ Chef de la Commission de Pisciculture des Bassins de ln Loire el de la Seine.
}

L'Union piscicole de France a organisé, du 12 au 25 décembre $19^{28}$, une exposition qui a eu lieu au Grand-Palais des Champs-Elysées, à paris, en mime temps que le $1 \mathrm{I}^{\mathrm{e}}$ Salon nautique international.

Cette manifestation, d'une ampleur dépassant celle des précédentes, a nécessité, en effet, un emplacement comprenant toute la galerie B (720 mq), la rolonde lui faisant suite ( $20 \mathrm{mq}$ ), enfin une partie de la grande nef ( $20 \mathrm{mq})$. Elle a été très réussie et il paraît utile d'en conserver le souvenir et d'en tirer quelque enseignement.

(1) Le lecteur pourra trouver des renseignements a ce sujet dans un travail public jar M. le Professeur Louis Rovlf et moi-meme dans les Annales de l'Institut Océanoyjaphique de Salambo (Annales $n^{\circ}$ III, 1927) et dans les ctudes de M. le Professeur louis Roune sur "Les Poissons et le monde vivant des caux "(tome II : La vie et l'action). 
C̈est ce que nous nous proposons de faice en donnant, d’abord, une description sommaire de l'Exposition piscicole et en indiquant, ensuite, les réflexions que nous a inspirées sa visite.

Cette lixposition élait divisée en trois sections : - celle des loissons vivants : - celle de la pèche fluviale et du matériel de pisciculture en eau douce ; - celle, enfin, des appareils faucardeurs.

Yous les passerons rapidement en revue, en insistant sur la première, à raison de son importance prépondérante.

$$
\text { I. - Polssons vivars (Galerie B) }
$$

La présentation des produits de l'élevagre du l'oisson d'eatu louce est lobjet essentiel d'une exposition comme celle organisée par l'Lnion piscicole. Il importe de mettre les produits en question sous les reux du public afin d'y recruter des clients.

On pratique trois sortes de culture - en viviers, en étangs on en aquariums - les deux premières donnant du Poisson de consommation, lat dernière le Poisson d'ornement. Sur cette base ont été établies trois sous-sections.

\section{A) Poissonts de viviers (Salmonides)}

Le simmon de fontaine, la Truite are-en-ciel et la Truite commune, qui sont les trois espèces avec lesquelles s'obtiennent les "portions" servies aux gourmets dans les hôtels ou restaurant, exigent des eaux limpides, fraîches, abondamment renouvelées. On ne rencontre pas partout les emplacements propices à leur élevage ; les réçions les plus favorisées, à cet éçard, sont l'Artois, la Normandie et la Picardie.

La plupart des propriétaires ou exploitants sont adhérents du Syndicat des pisciculleurs salmonicullears de France, un des groupements constitulifs de l'l nion piscicole de France. Son siège est a Paris, \&, rue d'Athènes et il esl actuellement présidé par M. Sornitez, qui fut l'un des membres du jury.

Lal sous-section des Poissons de viviers occupait 17 grands bacs de 1 mètre ou $1 \mathrm{~m}$. jo de longueur dans la gralerie $B$ du Grand-Palais.

Ceux portant les numéros a à 6 avaient élé attribués aux Piscicultures de Normandie, dont le directeur propriélaire est II. Bracmé, à Bernay (Eure).

On y remarquail : comme 'lruites arc-en-ciel :- - des reproducteurs de 3 ans (poids, r kil oio-r kil. 65o), - des alevins de 6 mois (poids, 3o40 gr.) ; - enfin des sujels de 16 mois sélectionnés (poids, 230-6ro gram.) issus de reproducteurs âgés de $\{$ ans prosenant doeufs importés de Californic en $192 \%$.

De nime, pour la Truile commune étaient exposés : - des reproducleurs de $6-7$ ans (poids, 3 kil. 625-4 kil. 700); - des alevins de a été (poids maximum, go gram.) ; - et des sujets de 2 élés (poids maximum, 260 gram.). 


\section{EXPOSITION DE PISCICULTURE DE PARIS (DÉCEMBRE 1928;}

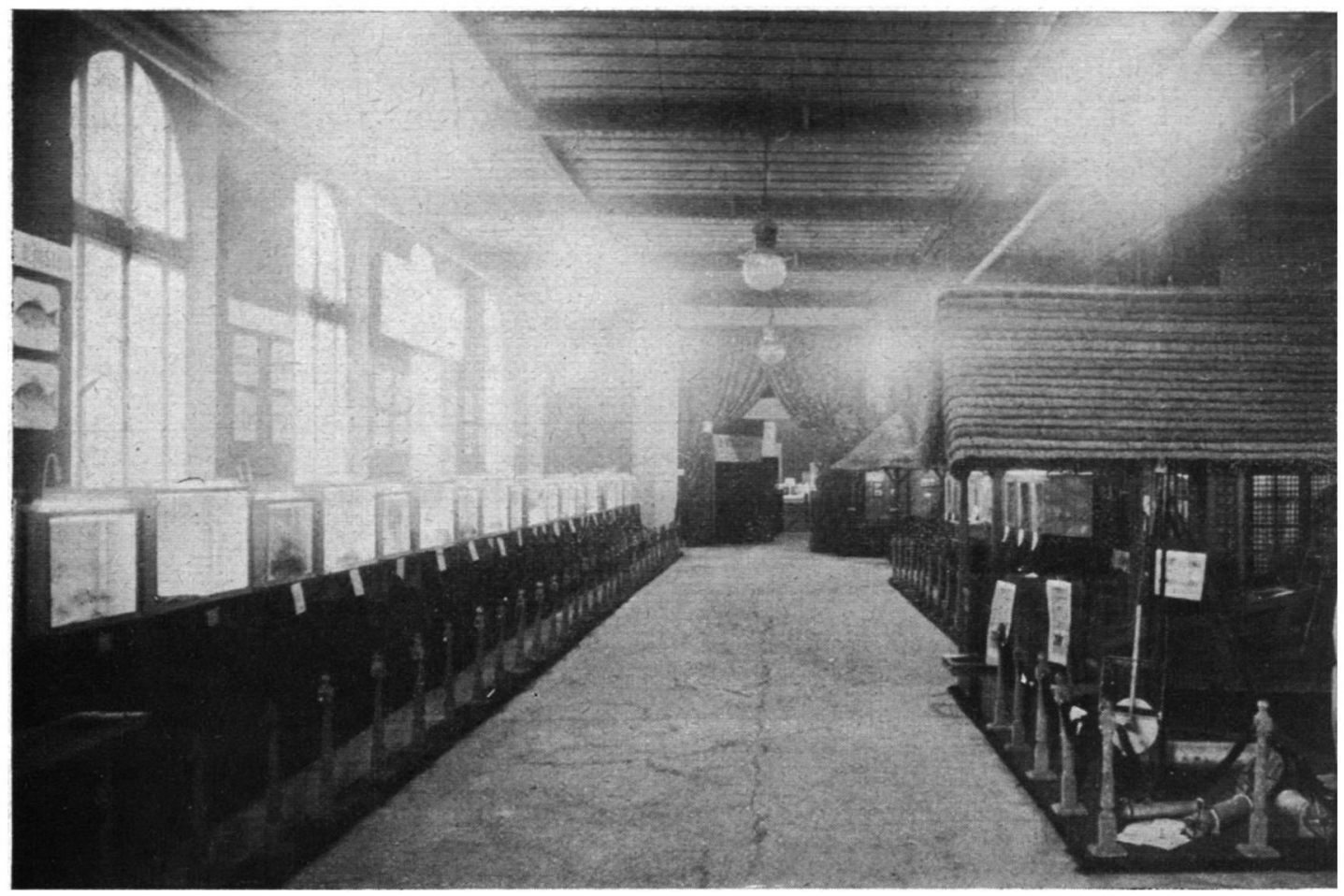

Fig. 4. - Vue d'ensemble (Galerie B).

(Cliché Nobécourr).
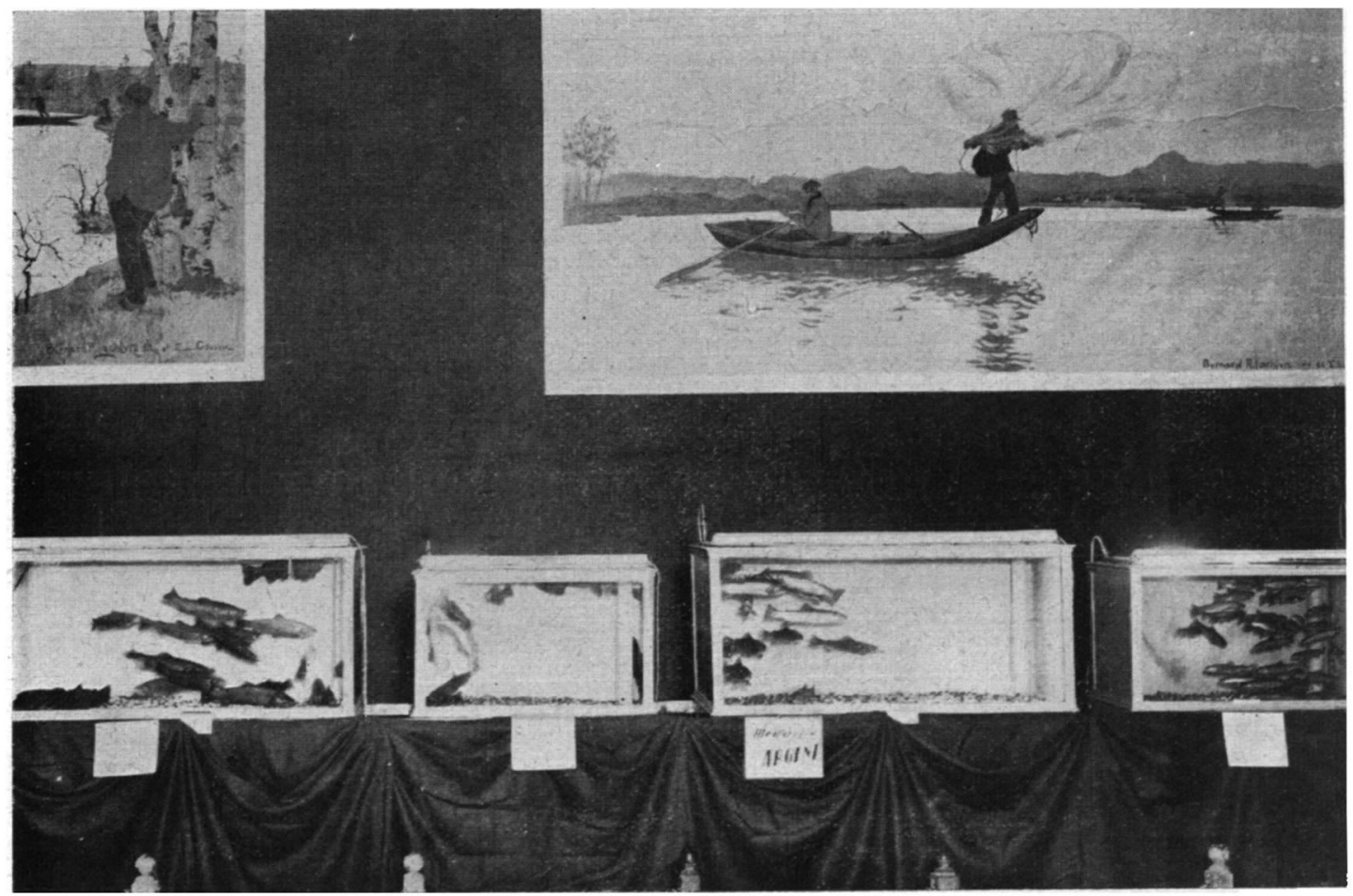

Fig. 5. - Quelques bacs (Galerie B). 
En outre, bien qu'on fût dans le rajon de la salmoniculture, un bac $\left(n^{\circ}\right.$ 4) hébergeait de grosses Carpes, souche Franconie, ainsi que des Tanches, ces deux espèces étant élevées par M. Bravmé à titre accessoire, comme consommant le superflu de la nourriture distribuée aux Salmonides.

A signaler, devant les bacs des Piscicultures de Normandie, ses cuviers de transport, en bois, avec diffuseurs d'oxygène, permeltant d'assurer une bonne livraison, à toute distance, de Poissons réputés délicats.

Il convient aussi de mentionner une artistique et vivante représentation d'une Truite en action de saut, sculpture sur bois, ouvre de M. IE Huis.

La Pisciculture de Vaucheron, à Gondrecourt (Meuse), avait peuplé les bacs 7 à rr. Propriété de Y. IInzur.rx, de Nancy, elle est dirigée par M. Mirite.

Sa spécialité est l'élevage du Saumon de fontaine dont elle présentait : - des reproducteurs de 4 ans (poids, I kil-1 kil. 50o), - des alevins de I été (poids, 6o-go gram.), - des sujets de 2 étés (poids, 400-600 gram.).

Comme. Truite arc-en-ciel, on remarquait :-- des reproducteurs de 4 ans (poids, I kil.- 1 hil. 5oo); - des alevins de 6 mois I/2 (taille, 9I/4 centt) ; - des sujets de z étés (poids, 300-40o gram.).

MM. Digny frères, 2o, quai du Louvre, Paris $\left(\mathrm{I}^{2 *}\right)$, possèdent les Salmonicultures d'Ilondouville (Eure) et des Vivrets-Marquéglise, par Ressonssur-Matz (Oise).

Ils exhibaient - outre les Poissons d'ornement, dont il sera question plus loin - dans le bac $n^{\circ} 13$, des Truites arc-en-ciel âgées de, 2 étés. Des alevins de 9 mois de cetle même espèce animaient un des aquariums de la rotonde $\left(\mathbf{n}^{\circ} 7 \mathrm{o}\right)$.

En outre, dans le bac $n^{\circ}$ r3 étaient présentées des $\mathbf{M}$ guilles provenant des étangs de Marquéglise.

Les deux piscicultures de Bouafles, par Vieux-Ronen-sur-Bresle (SeineInférieure) et du Prouzel, à Vers, par Saleux (Somme), ont comme propriétaire-directeur M. Monnorrer.

Ses Truites arc-en-ciel retenaient l'attention par leurs dimensions : reproducteurs de 6-7 ans (bac $n^{\circ} \mathrm{I} /$ ) : - alevins de 2.2 mois (bac $\mathbf{n}^{\circ} \mathbf{I} \overline{0}$ ). Ces derniers ont valu à leur éleveur le prix d'honneur du Ministère de l'Agriculture spécialement réservé, cette année, aux $\Lambda$ rc-en-ciel portions.

Les deux derniers bacs $\left(n^{05}\right.$ i 6 et 17$)$ attribués aux Poissons de viviers étaient occupés par les produits de la "Salmonide ", Société anonyme de pisciculture, à Offranville, par Saint-Aubin-sur-Scie (Seine-Inférieure), dont l'liministrateur délégué est M. Vioncfi-te-Duc, 68, rue Condorcet, Paris (IN). Cette Société élève le Saumon de fontaine et la Truite arc-en-ciel, représentés par des sujets ayant respectivement 23 mois et 6 mois $1 / 2$. 


\section{B) Poissons d'étang (Cyprinides)}

Exploitation d'étangs et carpiculture sont, en Europe, termes à peu près synonymes. On élève, en effet, surtout la Carpe dans les amas d'eaux artificiellement aménagés sur beaucoup de points dans les régions à sol imperméable, notamment, pour la France, en Brenne, Champagne, Dombes, Forez, Limousin, I.orraine et Sologne.

Plusieurs centaines d'éleveurs sont groupés en treize Syndicats fédérés par l'Union nationale des Syndicats de l'étang, avant son siège en l'Hôtel de la Société des Agriculteurs de France, \&, rue d'Athènes, Paris (IX ${ }^{\mathrm{e}}$ ). Le Président est, depuis le " janvier 19ag, M. Gat.ıce, successeur de M. Dexizer, en fonctions au moment de l'Exposition objet du présent compte rendu.

Entre les divers groupements affiliés à l'Union nationale se trouvaient répartis 95 bacs de $0 \mathrm{~m}$. 9o, I mètre et I $\mathrm{m}$. 5o de longueur, installés, comme ceux des Salmoniculteurs, dans la Galerie B du Grand-Palais.

Le Président du Syndicat des risciculteurs de Franche-Comté et de Bourgogne, Docteur Léculifr, à Champrougier, par Sellières (Jura), avait peuplé l'un d'eux $\left(\mathrm{n}^{\circ}{ }_{2} 3\right)$ de sujets de 2 ítés (poids moyen, 1 hil. zoo) de Carpe miroir, souche Galicie.

Le Syndicat des propriétaires d'étangs de la Dombes, présidé par M. Cinanvíriar, château de Romans, par Châtillon-sur-Chalaronne (Ain), était représenté par sa filiale, la Coopérative agricole des producteurs de Poissons de la Dombes. Cet organisme, de fondation récente, dont le siège est 14, quai de Retz, à Lyon et que dirige M. DF SArvr-Maurice, à Condessiat (Ain), avait envoyé à l'exposition des spécimens de Carpes marchandes, moitié type à écailles, moitié type cuir, avec spécimens d'alevins de 1 été et sujets de 2 étés. Ces Poissons, qu'accompagnaient des Tanches, occupaient les deux hacs $n^{\circ 8} 2 / 4$ et 25.

Ie Syndicat des propriétaires el exploitants des élangs du Forez a comme I'résident le Comte ji: Nrufiouns, chàteau de Reauvoir, par Boën-sur-Lignon (Loire).

Trois bacs $\left(\mathrm{n}^{\text {os }} 26\right.$ à 28$)$ contenaient des Carpes cuir, souche Wittingau, de forme trapue, soit reproducteurs, soit sujets de 2 étés. Ces derniers, dont certains exemplaires nés en Mai 1927, atteigrnaient, en Novembre 1928, des poids de $2 \mathrm{kil}$. $1 / 4$ à $2 \mathrm{kil}$. $1 / 2$, avec une moyenne de $2 \mathrm{kil}$. Ióo, ont reten i l'attention du jury qui attribua, à leur éleveur, une des deux médailles de vermeil accordées par le Ministre de l'Mgriculture, à titre de Prix d'honneur.

M. DE Neufboung avait, en outre, garni de Tanches le bac $n^{\circ}$ /1/.

Le Syndicat d'aquicullure dü Centre a à sa tète, depuis sa fonclation, M. M. Du Purtison, château de Vaựuenigge, par Saint-l'ardoux (HauteVienne).

Trois de sos adhérents avaient participé à l'cxposition. 
M. Lhíririen, à Ambazac (Haute-Vienne) présentait (bac $\mathbf{n}^{\circ}$ 29) des Carpes cuir, type commercial, ne présentant presque plus trace d'écaillure en sujets de 5 mois $1 / 2$ (poids, 15 -20 gram.).

M. Du Pưtrson avait peuplé de sujets variés le grand bac voisin $\left(n^{\circ} 30\right)$ où se trouvaient : - des reproducteurs de Carpe à écaille, de souche limousine, améliorée par $2 \%$ années de sélection; - des (arpes rouges (Hi-Gö̈) avec et sans écailles, -. enfin des Tanches de Mongolie, de teinte orangée, avec mouchetures, inléressantes comme Poissons d'ornement.

Le Comte de la Bastine, à Magnac-Bourg (Haute-Tiennc), avait envové des feuilles (I été) de Carpe miroir, souche Galicic.

Le Syndicat des propriétaires d'élangs de la Marne, présidé par M. Gallice, Pavillon de Grand-Fosse, par Saint-Martin d'Mblois (Marne), avait, lui aussi, trois élevages représentés.

D'abord celui de Boursault (1I. (intice) : - reproducteurs de Carpe miroir (bac $\mathrm{n}^{\circ} 32$ ).

Puis celui de Courville, par Fismes (Y. Yarooss), où se poursuit depuis six ans, la sćlection de la Carpe à ćcaille, souche champenoise ; on en voyait (bac $n^{\circ} 33$ ) des sujets d'un éló (poids, go gram.) et de 2 étés (poids, I kil. räo).

Enfin, le Comte de Vibraye (Pisciculture de Mareuil-en-Brie) avait envoyé des reproducteurs'de ('arpe à écailles, souche Lausitz.

Le Président du Syndicat des propriétaires d'étangs de la région du Nord-Ouesl, M. P'́xin de iA liabiène, présentuit les produits de ses étangs de Villebon, par Courville (Eure-et-Loir), dans le bac $n^{\circ} 46$, savoir Carpes à écailles, souche Wittingau, et Tanches.

Le Président de la Chambre syndicale des étangs dé Touraine et d'Anjou, M. Ilinscr, à la Iiouillardière, par Amboise (Indre-et-Loire), avait garni deux bacs avec des sujets élevés aux Jumeaux. Dans l'un $\left(n^{\circ} 35\right)$ figuraient des Tanches sonche tourangelle, sélectionnées, âgées de 3 étés ; - dans l'autre $\left(n^{2} 36\right)$ d'impośantes Ciarpes cuir, souche Wittingau, forme mi-longue à thorax épaissi.

(A suivre). 